\title{
Effectiveness of a Videoconferencing-Delivered Psychological Intervention for Mental Health Problems during COVID-19: A Proof-of-Concept Randomized Clinical Trial
}

\author{
Richard A. Bryant ${ }^{a, b, c}$ Katie S. Dawson ${ }^{a}$ Dharani Keyan $^{a}$ Suzanna Azevedo ${ }^{a}$ \\ Srishti Yadav ${ }^{a}$ Jenny Tran ${ }^{a}$ Natasha Rawson ${ }^{a}$ Samuel Harveya, c \\ aUniversity of New South Wales, Sydney, NSW, Australia; ${ }^{b}$ Westmead Institute for Medical Research, Sydney, NSW, \\ Australia; 'Black Dog Institute, University of New South Wales, Sydney, NSW, Australia
}

\section{Keywords \\ COVID-19 · Behaviour therapy · Controlled trial · Anxiety · Depression}

\begin{abstract}
Introduction: Anxiety and depression have increased markedly during the COVID-19 pandemic. There is a lack of evidence-based strategies to address these mental health needs during the pandemic. Objective: We aim to conduct a proof-of-concept trial of the efficacy of a brief group-based psychological intervention delivered via videoconferencing for adults in Australia distressed by the pandemic. Methods: In this single-blind, parallel, randomised controlled trial, adults who screened positive for COVID-related psychological distress across Australia were randomly allocated to either a 6-session group-based program based on behavioural principles $(n=120)$ or enhanced usual care (EUC, $n=120)$. Primary outcome was total score on the Hospital Anxiety and Depression (HADS) anxiety and depression subscales assessed at baseline, 1 week posttreatment, 2 months (primary outcome time point), and 6 months after treatment, as well as secondary outcome measures of worry, sleep impairment, anhedonia, mood, and COVID-19-related stress. Results: Between May 20, 2020, and October 20, 2020, 240 patients were enrolled into the trial. Relative to EUC, at 2 months par-
\end{abstract}

ticipants receiving intervention showed greater reduction on anxiety (mean difference, $1.4[95 \% \mathrm{Cl}, 0.3$ to 2.6$], p=0.01$; effect size, 0.4 [ $95 \% \mathrm{Cl}, 0.1$ to 0.7$]$ ) and depression (mean difference, $1.6[95 \% \mathrm{Cl}, 0.4$ to 2.8$], \mathrm{p}=0.009$; effect size, 0.4 [95\% $\mathrm{Cl}, 0.2$ to 0.7$])$ scales. These effects were maintained at 6 months. There were also greater reductions of worry, anhedonia, COVID-19-related fears, and contamination fears. Conclusions: This trial provides initial evidence that brief group-based behavioural intervention delivered via videoconferencing results in moderate reductions in common psychological problems arising during the COVID-19 pandemic. This program may offer a viable and scalable means to mitigate the rising mental health problems during the pandemic.

(c) 2021 The Author(s).

Published by S. Karger AG, Basel

\section{Introduction}

The COVID-19 pandemic has led to marked increases in mental health problems, with meta-analyses indicating a global prevalence estimate of $28.0 \%$ for depression and $26.9 \%$ for anxiety [1]. Longitudinal evidence suggests that

The trial was prospectively registered on Australian and New Zealand Clinical Trials Registry \#ACTRN12620000468921.

C 2021 The Author(s).

Published by S. Karger AG, Basel

This is an Open Access article licensed under the Creative Commons Attribution-NonCommercial-4.0 International License (CC BY-NC) (http://www.karger.com/Services/OpenAccessLicense), applicable to the online version of the article only. Usage and distribution for commercial purposes requires written permission.
Correspondence to:

Richard A. Bryant, r.bryant@ unsw.edu.au 
depression (standardized mean difference 0.67 ) and anxiety (standardized mean difference 0.40 ) have increased during the pandemic compared to 2019 [2]. The evidence indicates that fear of infection, loneliness during lockdowns, fear of shortage of essential supplies, and financial stress contribute to these psychological problems [3-5].

The rising mental health problems during the pandemic and the challenges of delivering mental health programs during lockdowns have led to calls for remote delivery of evidence-based services [6]. In response to this need, we adapted a program developed by the World Health Organization (Problem Management Plus; PM+) to reduce psychological distress in people affected by adversity $[7,8]$ which has been validated in multiple trials [9-11]. To address the specific concerns associated with the pandemic, the intervention was adapted to be a 6-session program that included strategies on (a) managing pandemic-related worries, (b) compensating for limited access to positive activities, and (c) accessing social supports during lockdown. The program was delivered to small groups (4 people) to facilitate greater efficiencies in delivery and utilized videoconferencing to overcome restrictions imposed by lockdown.

Here, we report a study of a randomized controlled trial of the intervention to reduce psychological distress during COVID-19. We hypothesized that the intervention would result in greater reductions in anxiety and depression, as well as reduced worry, sleep disturbance, positive and negative mood, anhedonia, and COVID-19 related concerns, relative to enhanced usual care (EUC).

\section{Methods}

\section{Trial Design}

The trial design has been previously described [12]. In this randomised, parallel, controlled trial, distressed people were randomly assigned to either the intervention or EUC on a 1:1 basis. Randomization was conducted by a researcher who was independent of the trial using computerised software to generate random number sequences with a block size of 4 , assigned participants, and kept a file of the assignments. The primary outcome time point was a 2-month follow-up after the intervention, with a secondary 6-month follow-up. Assessments were conducted online via a link emailed to participants by the trial coordinator, such that assessments were independent of any personnel involved in treatment condition allocation or delivery. The trial is reported according to the CONSORT guidelines [13].

Participants, Recruitment, and Inclusion/Exclusion Criteria

Participants were recruited between May 2020 to August 2020 following online advertising across Australia to participate in a trial to manage distress during COVID-19. People were invited to access the trial website, complete informed consent, and then complete the
General Health Questionnaire (GHQ-12 [14]). The GHQ-12 is a 12item self-report measure of psychological distress that is scored on a 4-point scale ranging from 0 to 3; the GHQ-12 can be scored dichotomously (i.e., 0-0-1-1), and when used as a screening tool the recommended cut-off score for elevated distress is $\geq 3$ [15]. Inclusion criteria included (a) adult (18 years or older), (b) score $\geq 3$ on the GHQ-12, and adequate English language comprehension. Exclusion criteria was determined by self-report on the online screening platform and included current psychosis, imminent suicidal risk, or current substance dependence, current psychotherapy, or no internet-based access for videoconferencing. Suicide risk was assessed with dichotomously answered questions, and participants were excluded if they endorsed suicidal plan or attempt in the past 6 months and referred to specialist services. Current psychotropic medication was permitted if the dosage was stable for 2 months.

\section{Interventions}

The adaptation, led by the senior author of PM+ (K.S.D.), was conducted over 8 weeks, and involved six focus groups with people affected by the pandemic who discussed the major mental health concerns during the pandemic and opinions about the intervention. The intervention comprised $6 \times 60$-min sessions that were delivered to groups of four participants on a videoconferencing platform by a Masters or Doctoral level clinical psychologist. The facilitators were trained by K.S.D. over 8 days, and each led a full practice group program under supervision from K.S.D. Prior to the group sessions, each participant in the treatment arm received an individual 10- to 15-min introductory online session to explain the program. In each session, instructional material was presented by brief pre-recorded webinars, which facilitators would use in group discussions. Session 1 comprised education about common reactions to COVID-19, including anxiety, depression, and worries, and also gave instructions in controlled breathing. Session 2 instructed participants to distinguish unhelpful worries from controllable problems by using a personalised worry decision tree, and how to identify achievable steps to manage problems. Session 3 focused on managing unhelpful worries, including setting up a "worry time" to limit time spent on worrying, strategies to manage intrusive worries that included mindfulness-based breathing, and revision of problem management strategies. Session 4 guided participants in behavioural activation strategies, focusing on engaging in pleasurable activities and those involving social connections; specific attention was given to achieving these steps in the context of lockdowns. Session 5 was devoted to reviewing strategies taught in the previous sessions via group discussions, and trouble-shooting barriers to implementing strategies. Session 6 focused on further revision of strategies, with a strong focus on relapse prevention. At each session, participants were given structured exercises to rehearse between sessions, and at each session the strategies taught in the previous sessions were reviewed (the full intervention manual is available at www.traumaticstressclinic.com).

The EUC arm comprised emailing participants a resource package comprising handouts detailing the strategies taught in the intervention. They were provided with instructions to work in a self-paced manner over 6 weeks. This comparator was employed because self-help coping strategies is a standard mental health outreach program offered during the COVID-19 pandemic (handout available in supplementary material).

To assess protocol adherence, sessions were recorded, and two independent psychologists rated $20 \%$ of sessions using a checklist. 
Fidelity was very high with $96 \%$ of rated sessions having perfect adherence to the protocol. Treatment quality was rated highly with a mean rating of 6.1 (possible range: $0-7$ ). Adverse reactions were monitored each session by group facilitators and by reviewing assessments; adverse events were referred to the Data Safety Monitoring Committee. All cases were followed up within $24 \mathrm{~h}$.

\section{Measures}

All participants who agreed to participate were emailed the link to the online assessment battery. This link was emailed at baseline, post-intervention, 2-month, and 6-month follow-up. Data were downloaded, and no researchers accessed the data prior to the final analysis. The outcome measures were selected to include patientreported outcome measures to reflect subjective experiences prior to and following the trial [16].

Primary Outcome. The primary outcomes were severity of anxiety and depressive symptoms measured using the Hospital Anxiety and Depression Scale (HADS [17]). The HADS is a 14 -item scale consisting of 2 subscales: HADS-A (anxiety, 7 items, range 0-21) and HADS-D (depression, 7 items, range 0-21). Higher scores indicate more anxiety and depression on each subscale, has been able to discriminate between those with and without each condition across many patient populations, and detects change in treatment trials $[18,19]$. A subscale score $\geq 8$ indicates probable caseness of anxiety and depression, respectively, and scores $\geq 15$ indicate severe levels of each condition [20]. To validate the selfreports of anxiety and depression on the HADS, clinicians who were unaware of the participants' self-reports on the HADS assessed 40 participants by clinical interview and estimated their HADS scores based on interview responses; the reliability between self-reported HADS and interview-based scores was 0.82 for depression and 0.88 for anxiety.

Secondary Outcomes. Worry using the Generalized Anxiety Disorder Scale (GAD-7 [21]), a 7-item self-report measure that possesses good psychometric properties, identifies people with severe worry, and is sensitive to change after treatment [22]. A cutoff score of 15 has been recommended to indicate generalized anxiety disorder [21]. Sleep difficulties were assessed using an adapted version of the Sleep Impairment Index (SII [23]), which is 5-item measure of problems in sleep onset, maintenance, early waking, disturbance caused by sleep problems, and distress caused by sleep problems. The SII has strong psychometric properties, is sensitive to treatment outcomes [24], and a cut-off of 10 is used to indicate insomnia [25]. Affect was assessed using the Positive and Negative Affect Schedule (PANAS [26]) on which participants describe their mood by rating 10 positive and 10 negative words; the PANAS has demonstrated good psychometric properties [26] and is widely used to detect affect change in affect after treatment [27]. Anhedonia was assessed using the Pleasure Scale that comprises 36 questions about a person's capacity to experience pleasure in various situations [28]; the scale has strong internal reliability and identifies depressed individuals with extreme anhedonia. To assess pandemic-related worries, the 36-item COVID Stress Scale [29] was used, which has 6 subscales that index (1) fears of the dangers of COVID-19, (2) contamination fears, (3) COVID19-related xenophobia, (4) fears of economic consequences of COVID-19, (5) traumatic stress, and (6) COVID-19-related checking. This recently published scale has yet to acquire strong clinimetric properties or demonstrated sensitivity to detect treatment outcomes.

Treating COVID-19 Anxiety and

Depression

\section{Statistical Analyses}

The sample size was calculated on the basis of prior trials of group-based $\mathrm{PM}+(11)$, which indicated that to achieve a moderate effect size of (0.5) a sample size of 105 participants would be needed per arm to provide power of 0.95 (alpha $=0.05$, two-sided); on the expectation that there would be approximately $10 \%$ attrition at the 2-month follow-up assessment, it was estimated that a total of 240 participants would need to be enrolled into the study.

Participant characteristics and potential differences between treatment completers and those who dropped out prior to the intervention period were analysed using planned $t$ tests for continuous measures and $\chi^{2}$ statistics for categorical variables; analysis of participant characteristics and those who were not retained at follow-up was subjected to an adjusted Bonferroni adjustment to accommodate multiple comparisons, resulting in an alpha level set at 0.005 . The primary analyses of outcome measures focused on intent-to-treat analyses. Hierarchical linear models were used to study differential effects of each treatment condition because this method allows the number of observations to vary between participants, which handles missing data by calculating estimates of trajectories using maximum likelihood estimation [30]. Fixed effects were tested for intervention condition and time of assessment. Fixed effects parameters were tested with the Wald test $(t$ test, $p<0.05$, two-sided) and 95\% confidence intervals. Analyses focus on the primary (HADS) and secondary (GAD-7, SII, PANAS, Pleasure Scale, and COVID Stress Scale) outcomes, with the main outcome timepoint being the 2-month follow-up. To assess if linear mixed models were biased by attrition of the sample, sensitivity analyses were conducted using only participants who completed the 2-month follow-up (the primary outcome time point). Following recommendations of understanding potential worsening of symptoms after an intervention (16), we calculated worsening of symptoms with the minimally important difference by comparing the proportions of participants showing a reduction of more than 0.5 SDs of the outcome measure [31]. To determine the effectiveness of the intervention on participants with more severe psychological disorders, we also conducted subgroup analyses focusing on participants who met the recommended cut-offs for severe anxiety or depression on the HADS [20] or for generalized anxiety disorder [21].

\section{Results}

\section{Baseline Characteristics of Participants}

Between May 20, 2020, and October 20, 2020 (with final 6-month follow-up assessment completed on May $28,2020) 240$ participants were enrolled into the trial. The majority of participants were female (83.8\%) and were university educated (64.6\%). In terms of caseness of psychological problems, $69.2 \%$ met the cut-off for probable depression, $90.4 \%$ for probable anxiety disorder, $65.4 \%$ for generalized anxiety disorder, and $43.8 \%$ for insomnia. In terms of severe disorder, $8.3 \%$ surpassed the cut-off for severe depression and $33.8 \%$ for severe anxiety. There were 120 participants randomized to the inter- 
Table 1. Demographic and participant characteristics

\begin{tabular}{|c|c|c|}
\hline & $\begin{array}{l}\text { Intervention } \\
(n=120)\end{array}$ & $\begin{array}{l}\text { Enhanced usual care } \\
(n=120)\end{array}$ \\
\hline Age, years & $37.3 \pm 12.8$ & $36.6 \pm 12.9$ \\
\hline Female sex, $n(\%)$ & $101(84.2)$ & $100(83.3)$ \\
\hline \multicolumn{3}{|l|}{ Employment status, $n(\%)$} \\
\hline Full-time & $41(34.2)$ & $52(43.3)$ \\
\hline Part-time & $25(20.8)$ & $21(17.5)$ \\
\hline Student & $28(23.3)$ & $22(18.3)$ \\
\hline Unemployed & $20(16.7)$ & $20(16.7)$ \\
\hline Retired & $5(4.2)$ & $5(4.2)$ \\
\hline \multicolumn{3}{|l|}{ Relationship status, $n(\%)$} \\
\hline Spouse & $62(51.7)$ & $57(47.5)$ \\
\hline Divorced/separated & $11(9.2)$ & $10(8.3)$ \\
\hline Single & $47(39.2)$ & $53(44.2)$ \\
\hline \multicolumn{3}{|l|}{ Education, $n(\%)$} \\
\hline High school & $19(15.8)$ & $23(19.2)$ \\
\hline Trade certificate & $11(9.2)$ & $12(10.0)$ \\
\hline Diploma & $6(5.0)$ & $14(11.7)$ \\
\hline University degree & $84(70.0)$ & $71(59.2)$ \\
\hline \multicolumn{3}{|l|}{ Ethnicity, $n(\%)$} \\
\hline Australian & $70(58.3)$ & $80(66.7)$ \\
\hline Asian & $16(13.3)$ & $11(9.2)$ \\
\hline European & $18(15.0)$ & $14(11.7)$ \\
\hline Other & $16(13.3)$ & $15(12.5)$ \\
\hline Probable depression, $n(\%)$ & $87(72.5)$ & $79(65.8)$ \\
\hline Severe depression, $n(\%)$ & $10(8.3)$ & $10(8.3)$ \\
\hline Probable anxiety, $n$ (\%) & $104(86.7)$ & $113(94.2)$ \\
\hline Severe anxiety, $n(\%)$ & $42(35.0)$ & $39(32.5)$ \\
\hline Generalized anxiety disorder, $n$ (\%) & $74(61.7)$ & $83(69.2)$ \\
\hline Insomnia, $n(\%)$ & $54(45.0)$ & $51(42.5)$ \\
\hline
\end{tabular}

Probable depression, HADS Depression Scale score $\geq 8$; Probable anxiety, HADS Anxiety Scale score $\geq 8$; Severe depression, HADS Depression Scale score $\geq 15$; Severe anxiety, HADS Anxiety Scale score $\geq 15$; Generalized anxiety disorder, GAD-7 score $\geq 15$; Insomnia, Sleep Impairment Index score $\geq 10$. vention and 120 to EUC. Participants in the intervention and EUC did not differ at baseline on any sociodemographic characteristics or baseline psychopathology measures (Table 1$)$. The mean $( \pm S D)$ number of intervention sessions attended was $5.3 \pm 1.2$, with 104 (86.7\%) participants attending at least five sessions. The primary outcome assessment at 2-months was conducted for 102 (85.0\%) participants in the intervention and $87(72.5 \%)$ in EUC. At 6 months, there were 103 (85.8\%) in the intervention and 79 in EUC (65.8\%) retained. The flowchart of participant recruitment and retention is reported in Figure 1. Participants who were and were not retained at the 2-month follow-up did not differ on baseline demographic characteristics; those who were not retained had higher scores on HADS: Anxiety and GAD-7 (see online supplementary Table 1).

\section{Primary Outcome}

At 2 months, participants in the intervention arm reported greater reductions in depression on the HADS (mean difference 1.6 [95\% CI, 0.4 to 2.8)], $p=0.009$ ) and anxiety on the HADS (1.4 [95\% CI, 0.3 to 2.6], $p=0.01$ ). These results indicated moderate effect sizes for depression (0.4 [95\% CI, 0.2 to 0.7$])$ and anxiety (0.4 [95\% CI, 0.1 to 0.7$]$ ) (Table 2). At 2 months (for participants who completed this assessment), fewer participants in the intervention arm than those in EUC met the cut-off for depression ( 39.2 vs. $57.5 \%$, odds ratio 0.5 [ $95 \%$ CI, 0.3 to $0.9], p<0.02)$ and anxiety (62.7 vs. $78.2 \%$, OR 0.5 [95\% CI, 0.3 to 0.9 ], $p<0.03$ ) caseness. The number needed to treat was 5.5 for depression, and 6.2 for anxiety. In terms of severe caseness at follow-up, fewer participants in the intervention arm than those in EUC met the cut-off for severe anxiety ( 6.9 vs. $20.7 \%$, OR 0.3 [ $95 \%$ CI, 0.1 to 0.7 ],
Bryant/Dawson/Keyan/Azevedo/Yadav/ Tran/Rawson/Harvey 
Fig. 1. Study flow diagram.

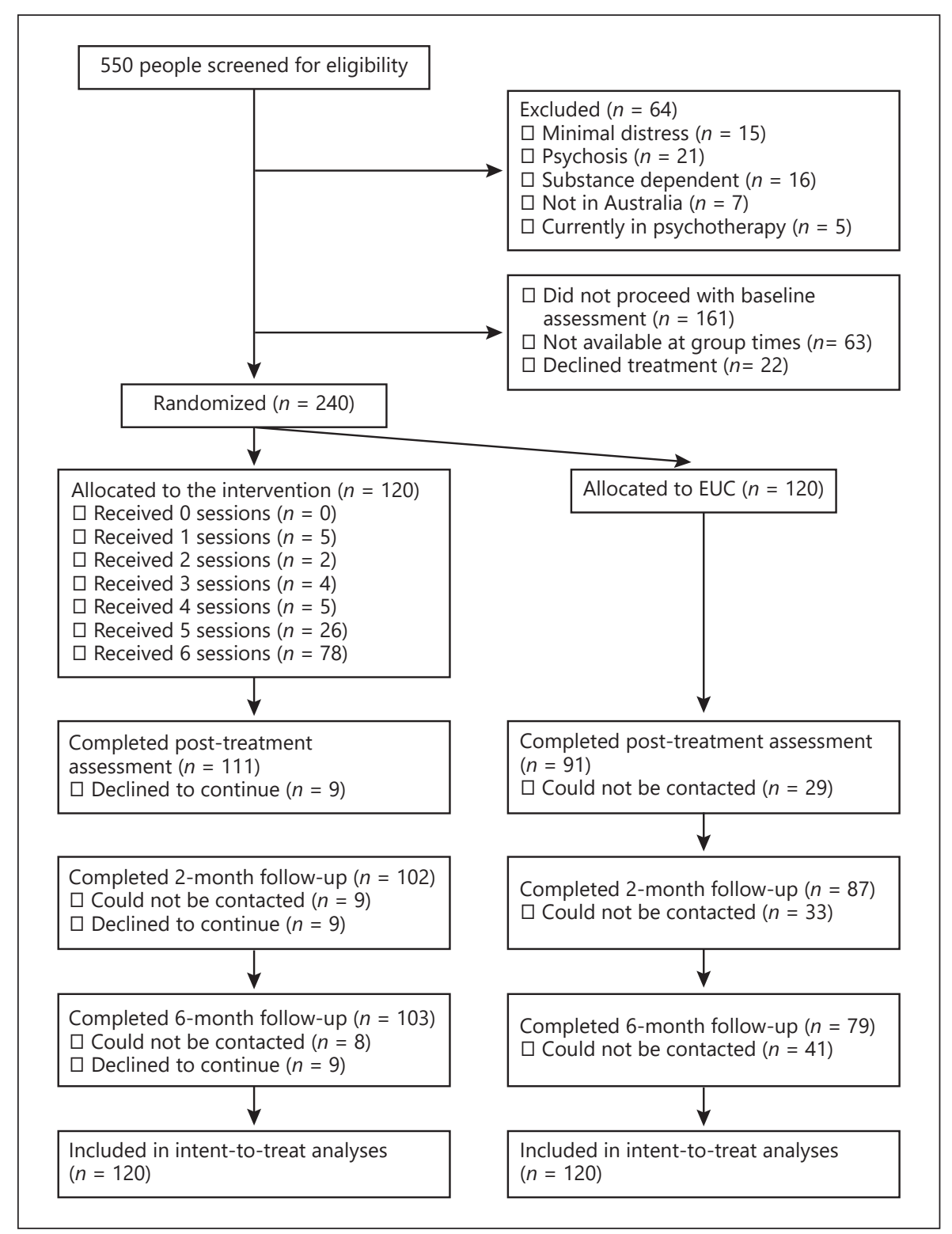

$p<0.01$ ) but not severe depression ( 4.9 vs. $6.9 \%$, OR 0.7 [95\% CI, 0.2 to 2.4$], p<0.6$ ). There were marginally more participants in the EUC arm than the intervention who reported worsening depression at the 2-month assessment ( 26.4 vs. $15.7 \%, p=0.07$ ), and more EUC participants reported worsening anxiety than those in the intervention $(16.1$ vs. $9.8 \%, p<0.05)$.

\section{Secondary Outcomes}

In terms of worry, the intervention led to greater reduction difference at 2-month follow-up (mean difference 1.6 [ $95 \% \mathrm{CI}, 0.2$ to 2.9 ], $p=0.02$ ), indicating a small effect size ( 0.3 [ $95 \%$ CI, 0.0 to 0.7] (Table 2). The interven- tion led to greater reduction in anhedonia (mean difference -9.0 [95\% CI, -14.7 to -3.4$], p=0.002$ ), indicating a small effect size $(-0.4$ [95\% CI, -0.7 to -0.2$])$. The intervention also resulted in greater improvement in positive mood of the PANAS (mean difference -4.1 [95\% CI, -6.4 to 1.9$], p=0.001)$, indicating a moderate effect size $(-0.6$ [95\% CI, -1.0 to 0.3$]$ ). The intervention led to greater reduction in negative mood of the PANAS (mean difference 2.7 [ $95 \% \mathrm{CI}, 0.5$ to 5.0 ], $p=0.02$ ), indicating a moderate effect size ( 0.3 [ $95 \% \mathrm{CI}, 0.1$ to 0.6$]$ ). In terms of COVID-19 related concerns, the intervention demonstrated greater reductions in danger of fear of pandemic dangers (mean difference 1.8 [95\% CI, 0.2 to 3.5], $p=0.03$ ), indi- 
Table 2. Summary statistics and results from mixed model analysis of primary and secondary outcomes

\begin{tabular}{|c|c|c|c|c|c|c|}
\hline \multirow{2}{*}{$\begin{array}{l}\text { Primary and secondary } \\
\text { outcomes }\end{array}$} & \multirow[t]{2}{*}{ Visit } & \multicolumn{2}{|l|}{ Descriptive statistics } & \multicolumn{3}{|l|}{ Mixed model analysis } \\
\hline & & $\begin{array}{l}\text { intervention } \\
(n=120) \\
\text { estimated mean }(95 \% \mathrm{Cl})\end{array}$ & $\begin{array}{l}\text { EUC } \\
(n=120) \\
\text { estimated mean }(95 \% \mathrm{Cl})\end{array}$ & $\begin{array}{l}\text { difference in LS mean } \\
(95 \% \mathrm{Cl})\end{array}$ & $p$ value & $\begin{array}{l}\text { effect size }{ }^{a} \\
(95 \% \mathrm{Cl})\end{array}$ \\
\hline \multirow[t]{4}{*}{ HADS-Depression } & Baseline & 9.7 (9.0 to 10.3 ) & 9.4 (8.8 to 10.1$)$ & & & \\
\hline & 7-week & $7.4(6.7$ to 8.1$)$ & 8.3 (7.5 to 9.1$)$ & 1.1 (0.1 to 2.2$)$ & 0.03 & 0.3 (0.0 to 0.6$)$ \\
\hline & 2-month & 7.1 (6.3 to 7.9) & 8.4 (7.6 to 9.3$)$ & 1.6 (0.4 to 2.8$)$ & 0.009 & $0.4(0.1$ to 0.7$)$ \\
\hline & 6-month & $6.4(5.6$ to 7.2$)$ & 7.5 (6.6 to 8.4$)$ & $1.3(0.8$ to 2.6$)$ & 0.04 & $0.4(0.2$ to 0.7$)$ \\
\hline \multirow[t]{4}{*}{ HADS-Anxiety } & Baseline & 12.6 (11.9 to 13.3$)$ & $12.8(12.1$ to 13.5$)$ & & & \\
\hline & 7-week & $9.4(8.6$ to 10.3$)$ & $11.0(10.1$ to 11.8$)$ & $1.4(0.3$ to 2.4$)$ & 0.009 & $0.4(0.1$ to 0.6$)$ \\
\hline & 2-month & 9.1 (8.3 to 9.9 ) & 10.8 (10.0 to 11.7$)$ & $1.4(0.3$ to 2.6$)$ & 0.01 & $0.4(0.1$ to 0.7$)$ \\
\hline & 6-month & 9.2 (8.4 to 10.0$)$ & $11.0(10.1$ to 11.8$)$ & 1.5 (0.4 to 2.7$)$ & 0.01 & $0.4(0.1$ to 0.7$)$ \\
\hline \multirow[t]{4}{*}{ GAD-7 } & Baseline & $11.4(10.5$ to 12.2$)$ & 12.1 (11.2 to 12.9$)$ & & & \\
\hline & 7-week & 6.8 (5.9 to 7.7$)$ & 10.0 (9.1 to 11.0$)$ & 2.6 (1.4 to 3.7$)$ & 0.001 & 0.5 (0.3 to 0.8$)$ \\
\hline & 2-month & 6.8 (5.9 to 7.7$)$ & 9.1 (8.1 to 10.0$)$ & $1.6(0.2$ to 2.9$)$ & 0.02 & $0.3(0.0$ to 0.7$)$ \\
\hline & 6-month & 7.3 (6.4 to 8.2$)$ & 8.6 (7.5 to 9.6$)$ & $0.5(-.9$ to 2.0$)$ & 0.46 & $0.1(-0.2$ to 0.4$)$ \\
\hline \multirow[t]{4}{*}{ Sleep Impairment Index } & Baseline & 8.6 (7.8 to 9.4$)$ & 9.0 (8.2 to 9.8$)$ & & & \\
\hline & 7-week & 6.7 (5.9 to 7.5$)$ & 8.0 (7. to 8.8$)$ & $0.9(-0.1$ to 1.9$)$ & 0.07 & $0.2(-0.0$ to 0.5$)$ \\
\hline & 2-month & 6.5 (5.6 to 7.3$)$ & 7.5 (6.7 to 8.4$)$ & $0.7(-0.4$ to 1.8$)$ & 0.20 & $0.2(-0.1$ to 0.4$)$ \\
\hline & 6-month & $6.6(5.8$ to 7.4$)$ & 7.6 (6.7 to 8.5$)$ & $0.6(-0.6$ to 1.8$)$ & 0.31 & $0.2(-0.1$ to 0.4$)$ \\
\hline \multirow[t]{4}{*}{ PANAS-positive } & Baseline & 20.2 (18.9 to 21.5 ) & 20.6 (19.3 to 22.0$)$ & & & \\
\hline & 7-week & 23.7 (22.3 to 25.0$)$ & 21.4 (19.9 to 22.9$)$ & $-2.7(-4.7$ to -0.7$)$ & 0.008 & $-0.4(-0.7$ to -0.1$)$ \\
\hline & 2-month & 24.2 (22.8 to 25.6$)$ & 20.5 (19.0 to 22.0 ) & $-4.1(-6.4$ to 1.9$)$ & 0.001 & $-0.6(-1.0$ to 0.3$)$ \\
\hline & 6-month & 24.2 (22.8 to 25.6$)$ & 20.9 (19.3 to 22.5 ) & $-2.7(-4.6$ to -0.7$)$ & 0.008 & $-0.4(-0.7$ to -0.1$)$ \\
\hline \multirow[t]{4}{*}{ PANAS-negative } & Baseline & $23.6(22.2$ to 25.0$)$ & $23.8(22.4$ to 25.2$)$ & & & \\
\hline & 7-week & 17.1 (15.7 to 18.5$)$ & 21.5 (19.9 to 23.0$)$ & $4.1(2.1$ to 6.2$)$ & 0.001 & 0.5 (0.3 to 0.8$)$ \\
\hline & 2-month & 17.2 (16.1 to 19.1$)$ & 20.2 (18.6 to 21.8 ) & $2.7(0.5$ to 5.0$)$ & 0.02 & $0.3(0.1$ to 0.6$)$ \\
\hline & 6-month & $17.6(16.1$ to 19.1$)$ & 20.4 (18.7 to 22.0$)$ & $2.5(-3.6$ to 0.7$)$ & 0.03 & $0.3(-0.4$ to 0.1$)$ \\
\hline \multirow[t]{4}{*}{ Anhedonia } & Baseline & $126.4(122.2$ to 128.7$)$ & $124.50(120.3$ to 128.7$)$ & & & \\
\hline & 7-week & $133.0(128.7$ to 137.2$)$ & 124.8 (120.3 to 129.3$)$ & $-6.3(-11.5$ to -1.0$)$ & 0.02 & $-0.3(-0.5$ to 0.0$)$ \\
\hline & 2-month & 133.1 (128.8 to 137.5$)$ & 122.2 (117.6 to 126.8$)$ & $-9.0(-14.7$ to -3.4$)$ & 0.002 & $-0.4(-0.7$ to -0.2$)$ \\
\hline & 6-month & 129.3 (125.0 to 133.7$)$ & $121.4(116.7$ to 126.2$)$ & $-6.0(-11.8$ to -0.2$)$ & 0.04 & $-0.3(-0.6$ to 0.0$)$ \\
\hline \multirow[t]{4}{*}{ CSS: Danger } & Baseline & $11.9(10.8$ to 13.1$)$ & 10.8 (9.6 to 11.9$)$ & & & \\
\hline & 7-week & $9.4(8.3$ to 10.6$)$ & $9.9(8.7$ to 11.1$)$ & $1.6(0.1$ to 3.1$)$ & 0.03 & 0.2 (0.0 to 0.5$)$ \\
\hline & 2-month & $6.8(5.6$ to 7.9$)$ & 7.4 (6.2 to 8.7$)$ & $1.8(0.2$ to 3.5$)$ & 0.03 & $0.3(0.0$ to 0.5$)$ \\
\hline & 6-month & 5.7 (4.5 to 6.9$)$ & 7.1 (5.8 to 8.4$)$ & $2.5(0.8$ to 4.3$)$ & 0.004 & $0.4(0.1$ to 0.6$)$ \\
\hline \multirow[t]{4}{*}{ CSS: Economic Fears } & Baseline & 4.0 (3.3 to 4.8$)$ & 4.1 (3.4 to 4.8 ) & & & \\
\hline & 7-week & $2.8(2.0$ to 3.5$)$ & $3.3(2.5$ to 4.1$)$ & $0.5(-0.5$ to 1.4$)$ & 0.33 & $0.1(-0.1$ to 0.3$)$ \\
\hline & 2-month & $1.6(0.8$ to 2.4$)$ & $2.0(1.2$ to 2.9$)$ & $0.4(-0.8$ to 1.6$)$ & 0.50 & $0.1(-0.2$ to 0.3$)$ \\
\hline & 6-month & $1.3(0.5$ to 2.1$)$ & $2.0(1.2$ to 2.9$)$ & 0.7 (-0.6 to 2.0$)$ & 0.28 & $0.2(-0.1$ to 0.4$)$ \\
\hline \multirow[t]{4}{*}{ CSS: Xenophobia } & Baseline & 2.9 (2.1 to 3.7$)$ & 2.7 (1.9 to 3.5$)$ & & & \\
\hline & 7-week & 2.1 (1.3 to 2.9$)$ & 2.5 (1.7 to 3.4$)$ & $0.7(-0.4$ to 1.7$)$ & 0.23 & $0.1(-0.1$ to 0.3$)$ \\
\hline & 2-month & $1.4(0.5$ to 2.2$)$ & 2.7 (1.78 to 3.6$)$ & 1.5 (0.3 to 2.8$)$ & 0.01 & 0.3 (0.1 to 0.6$)$ \\
\hline & 6-month & 1.8 (1.0 to 2.6$)$ & 2.2 (1.3 to 3.2 ) & 0.7 (-.6 to 2.0$)$ & 0.32 & $0.1(-0.1$ to 0.4$)$ \\
\hline \multirow[t]{4}{*}{ CSS: Traumatic Stress } & Baseline & 7.5 (6.6 to 8.4$)$ & 6.8 (5.9 to 7.7$)$ & & & \\
\hline & 7-week & $5.1(4.2$ to 6.0$)$ & $5.4(4.5$ to 6.4$)$ & $1.0(-0.1$ to 2.2$)$ & 0.07 & $0.2(0.0$ to 0.4$)$ \\
\hline & 2-month & 3.2 (2.3 to 4.2$)$ & 3.7 (2.7 to 4.7$)$ & $1.1(-0.2$ to 2.5$)$ & 0.10 & $0.2(0.0$ to 0.5$)$ \\
\hline & 6-month & $2.4(1.5$ to 3.3$)$ & 3.0 (1.9 to 4.0$)$ & $1.3(-.2$ to 2.7$)$ & 0.08 & 0.2 (0.0 to 0.5$)$ \\
\hline
\end{tabular}


Table 2 (continued)

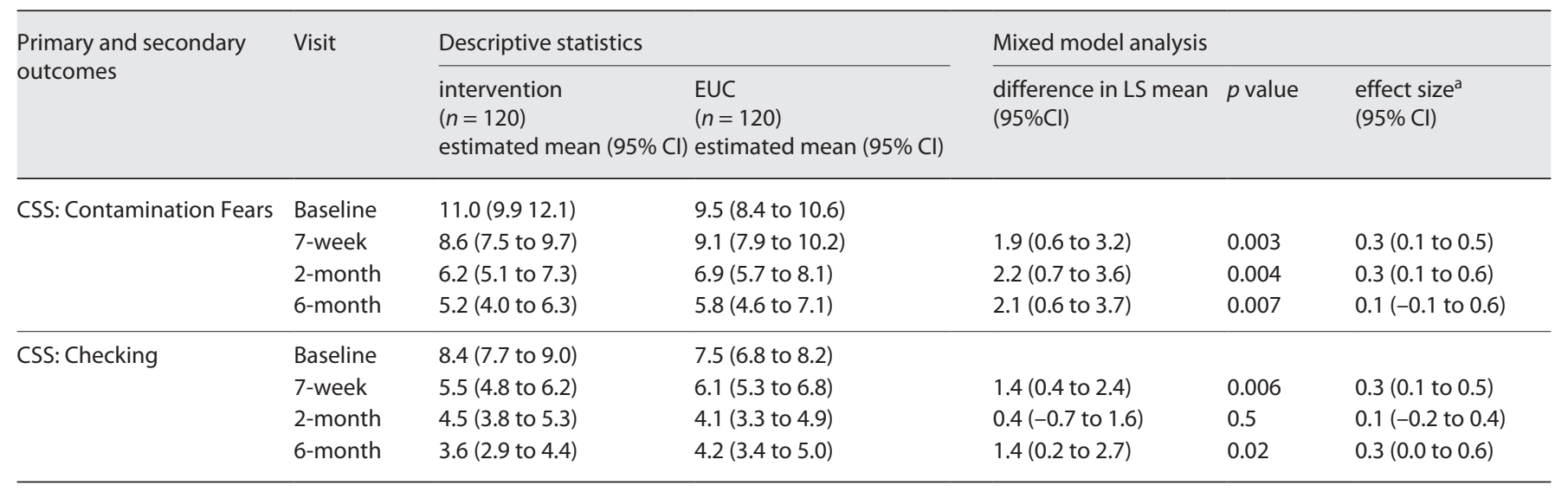

EUC, enhanced usual care; LS, least square; HADS, Hospital Anxiety and Depression Scale (depression subscale score range: 0-21; anxiety subscale score range: $0-21$; higher scores indicate elevated anxiety or depression); GAD-7 (total score range: 0-21; higher scores indicate more severe worry); SII, Sleep Impairment Index (total score range: 0-20; higher scores indicate more severe sleep impairment); PANAS, Positive and Negative Affect Schedule (subscale total score range: $10-50$ on positive and negative scales, respectively; higher scores indicate more greater positive and negative mood, respectively); Pleasure Scale (total score range: 36-180; lower scores indicate more severe anhedonia); CSS, COVID-19 Stress Scale (each scale total score range: 0-24; higher scores indicate more severe stress). Effect size was calculated by the difference in least square means between intervention and EUC from mixed model divided by the pooled standard deviation.

cating a small effect size (0.3 [95\% CI, 0.0 to 0.5$])$. The intervention achieved greater reductions in xenophobic attitudes (mean difference 1.5 [95\% CI, 0.3 to 2.8 ], $p=$ $0.01)$, indicating a small effect size $(0.3$ [ $95 \% \mathrm{CI}, 0.1$ to $0.6])$. There was also a greater reduction in contamination fears (mean difference 2.2 [95\% CI, 0.7 to 3.6], $p=$ $0.004)$, indicating a small effect size ( 0.3 [95\% CI, 0.1 to $0.6]$ ). The intervention and EUC had proportionally few participants deteriorating at 2-month assessment for worry (10.8 vs. $12.5 \%)$, sleep disturbance (19.4 vs. $24.4 \%$ ), positive affect (16.7 vs. $23.0 \%)$, negative affect (12.7 vs. $13.8 \%$ ), anhedonia (12.7 vs. $26.7 \%)$, fear of pandemic dangers ( 8.8 vs. $9.3 \%$ ), xenophobia ( 8.8 vs. $10.5 \%), 9.8$ vs. $7.0 \%)$, contamination fears ( 2.9 vs. $8.1 \%)$, checking (4.0 vs. $9.3 \%)$.

\section{Subgroup Analyses}

There were 164 participants who reported severe anxiety, severe depression, or generalized anxiety disorder at baseline; these were equally distributed between the intervention $(76,63.3 \%)$ and EUC (88 (73.3\%). In terms of analyses that focused on these participants (see online supplementary Table 2), the intervention resulted in greater reductions at 2-month follow-up on both anxiety (mean difference 2.2 [95\% CI, 0.7 to 3.7], $p=0.004$ ), indicating a moderate effect size (0.6 [95\% CI, 0.2 to 1.0$]$ ), and depression (mean difference 1.8 [95\% CI, 0.2 to 3.5], $p=0.03)$, indicating a large effect size $(0.5$ [95\% CI, 0.1 to
0.7]). Further, there were greater increases in positive mood, and decreases in worry, negative mood, anhedonia, xenophobia, and contamination fears in the intervention arm relative to EUC.

\section{Sensitivity Analyses}

The sensitivity analysis that focused only on participants who completed the 6-month follow-up indicated that in terms of primary outcomes, the intervention led to greater reductions than EUC for both depression and anxiety (see online supplementary Table 3 ). Similarly, for the secondary outcomes the intervention led to better positive mood, and greater reductions in worry, negative mood, anhedonia, and contamination fears, relative to EUC.

\section{Longer-Term Effects}

At 6 months, there were greater reductions on the primary outcomes of depression (mean difference 1.3 [95\% CI, 0.8 to 2.6$)], p=0.04)$, indicating a moderate effect size ( 0.4 [ $95 \%$ CI, 0.2 to 0.7$])$, and anxiety (1.5 [95\% CI, 0.4 to 2.7], $p=0.01)$, indicating a moderate effect size (0.4 [95\% CI, 0.1 to 0.7$]$ ). In terms of secondary outcomes, the intervention led to better positive mood, and greater reductions in negative mood, fear of pandemic dangers, contamination fears, and checking concerns (Table 2).

There were no adverse events throughout the trial. 


\section{Discussion}

This trial represents initial evidence that a groupbased psychological intervention utilizing videoconferencing can reduce levels of depression, anxiety, worry, sleep impairment, anhedonia, and COVID-19 related concerns. Although the current finding that the intervention can reduce psychological distress accords with previous studies of $\mathrm{PM}+$ that common mental disorders can be alleviated with a brief, transdiagnostic intervention [9$11]$, this program differs from prior trials because it was specifically adapted to meet the mental health needs of people during the pandemic. By providing the intervention via a videoconferencing platform, the trial showed that this intervention can be effectively delivered during lockdowns. Further, the observation that $86.7 \%$ of participants in the intervention attended at least 5 of the 6 scheduled sessions reflects the general acceptability of this program and provides an encouraging indication about this form of delivery for people distressed during the pandemic.

In the context of marked increases in common mental disorders in the wake of the pandemic $[32,33]$, this finding indicates this videoconferencing-based program may represent a viable and scalable option to address increased levels of distress and to prevent these mental health problems progressing to long-term mental ill health. The COVID-19 pandemic has created a surge in psychological distress globally [34]. Most existing mental health interventions available to countries experiencing this increase in distress are neither designed to address the specific symptoms generated by a pandemic nor are they able to be delivered at scale during ongoing lockdowns. Numerous commentators have noted that the unprecedented needs triggered by the pandemic require new applications of technology-driven mental health programs [6]. Online, telehealth, and videoconferencing programs are indicated for a number of reasons. First, the widespread locations of rising mental health problems across countries requires that remote delivery of mental health services need to be evaluated to reach people in diverse regions. Second, the fact that many people who are experiencing anxiety or depression are those who have not experienced these problems before [35] suggests that mental health services need to be easier for these people to access because they may not be inclined to attend mainstream mental health services. Third, programs such as this brief group intervention have the potential to be scaled up to large populations in a cost-effective manner, although we note that cost-effectiveness analysis is yet to be conducted on this program. The current study builds on an earlier trial conducted during the pandemic that found that a fully online program was efficacious in reducing worry one month after the program [36]. The present trial extends this earlier work by (a) demonstrating reductions in anxiety, depression, and worry, (b) showing that the gains could be maintained six months after treatment, and (c) comparing the program against a more active control than the waitlist used in the online program trial. The brevity of this program, its group format and videoconferencing delivery mean it can be implemented rapidly to large numbers of people. Our results demonstrate that this type of rapid response to emerging distress can help alleviate the predicted longer term public mental health consequences from the current pandemic. The finding that the benefits can persist 6 months after treatment indicate that the clinical gains are long-lasting. This intervention can be understood in the context of evidence that online treatments for anxiety and depression can effectively reduce these common mental health problems [37] and offer a viable alternative to face-to-face therapy.

The benefits achieved by the intervention were also observed when analyses focused on those with more severe anxiety, depression, or generalized anxiety disorder. The ability of the intervention to alleviate the severity of psychological distress in those with even more severe mental health problems underscores the potential utility of the intervention to assist the many people who are suffering psychological disorders as a result of the pandemic. The proven effectiveness of $\mathrm{PM}+$ to be delivered by lay providers $[9,10]$ suggests that non-specialists may be engaged to deliver the current intervention, which offers the opportunity for poorly resourced countries that lack sufficient mental health specialists to provide mental health assistance during the pandemic.

Relevant to the issues specifically arising during the pandemic, the intervention led to greater reductions than EUC in terms worry, fear of the dangers of COVID-19, anxiety about sources of contamination, and xenophobia. Whilst health anxiety is often present in a proportion of people, recent commentaries have noted that the nature of the pandemic has heightened this form of anxiety and preoccupation with illness [38]. The capacity of the intervention to reduce these pandemic-related concerns is important because worry and excessive fear of contamination and the capacity to be safe during the pandemic can contribute to additional medical and social problems as people engage in avoidance and are re-
Bryant/Dawson/Keyan/Azevedo/Yadav/ Tran/Rawson/Harvey 
luctant to seek medical care [39]. The observation that the intervention reduced xenophobic attitudes is also relevant because of evidence from previous pandemics that racial discrimination is expected to increase against targeted ethnic groups suspected of carrying the virus or attributing responsibility of the virus to these groups [40].

We note several study limitations. First, the participants in this trial were predominantly female and university educated; the results need to be replicated with a broader representation of the population. Second, although the trial was conducted in Australia during periods of severe lockdown, the rates of COVID-19 infections, hospitalizations, and deaths were much lower than those in many other countries. It will be important to evaluate the findings in countries with greater infection rates. Third, to facilitate the rapid conduct of this trial all assessments were conducted online via selfreport, and so the trial lacks structured clinical interviews. Our validation checks did indicate, however, that clinical ratings of anxiety and depression on the HADS supported the accuracy of the self-report measure. Fourth, the comparator in this trial was a self-help manual comprising the same skills taught in the group program because self-guided coping guides are the predominant mental health strategy being disseminated during the pandemic. We recognise that this comparator does not control for therapist attention or group involvement.

In summary, this intervention delivered in 6-session group format on a videoconferencing platform is able to reduce anxiety, depression, and other common psychological problems reported by many people during the COVID-19 pandemic. This program may offer a scalable and effective mental health intervention that can be delivered safely in the context of lockdowns and addresses many of the major psychological problems being experienced during the pandemic. These initial findings should be replicated by comparing the intervention against a more active control condition.

\section{Acknowledgement}

We are grateful to Phillipa Specker and Emma Bryant for assistance in treatment fidelity checks of therapy sessions.

\section{Statement of Ethics}

All of the participants provided informed consent while applying for participation in the study. This study was approved by the University of New South Wales Human Research Ethics Committee and conducted in accordance with the World Medical Association Declaration of Helsinki.

\section{Conflict of Interest Statement}

The authors have no conflicts of interests to declare.

\section{Funding Sources}

This research was funded by a National Health and Medical Research Council Investigator (1173921). The study funders had no role in the design and conduct of the study; collection, management, analysis, and interpretation of the data; preparation, review, or approval of the manuscript; and decision to submit the manuscript for publication.

\section{Author Contributions}

Study concept and design: R.A.B., K.S.D., D.K., S.A., S.Y., N.R., J.T., S.H. Data acquisition: K.S.D., D.K., S.A., S.Y., N.R., J.T. Data management: J.T. Analysis and interpretation of data: R.A.B., S.H. Drafting of manuscript: R.A.B., K.S.D., D.K., S.A., S.Y., N.R., J.T., S.H. Critical revision of manuscript for important intellectual content: R.A.B., K.S.D., D.K., S.A., S.Y., J.T., N.R., S.H. Statistical analysis: R.A.B. Obtained funding: R.A.B., S.H. Study supervision: R.A.B., K.S.D.

\section{Data Availability Statement}

The datasets generated and analysed during the current study are available at doi 10.6084/m9.figshare.14828304.

\section{References}

1 Nochaiwong S, Ruengorn C, Thavorn K, Hutton B, Awiphan R, Phosuya C, et al. Global prevalence of mental health issues among the general population during the coronavirus disease-2019 pandemic: a systematic review and meta-analysis. Sci Rep. 2021;11(1):10173.

2 Kunzler AM, Röthke N, Günthner L, StoffersWinterling J, Tüscher $\mathrm{O}$, Coenen $\mathrm{M}$, et al. Mental burden and its risk and protective fac- tors during the early phase of the SARSCoV-2 pandemic: systematic review and meta-analyses. Global Health. 2021;17(1):34.

3 Wang Y, Kala MP, Jafar TH. Factors associated with psychological distress during the coronavirus disease 2019 (COVID-19) pandemic on the predominantly general population: a systematic review and meta-analysis. PLoS One. 2020;15(12):e0244630.
4 Killgore WDS, Cloonan SA, Taylor EC, Dailey NS. Loneliness: a signature mental health concern in the era of COVID-19. Psychiatry Res. 2020;290:113117.

5 Zhang SX, Wang Y, Jahanshahi AA, Li J, Schmitt VGH. Early evidence and predictors of mental distress of adults one month in the COVID-19 epidemic in Brazil. J Psychosom Res. 2021;142:110366.
Treating COVID-19 Anxiety and Depression
Psychother Psychosom 2022;91:63-72 DOI: $10.1159 / 000520283$ 
6 Wright JH, Caudill R. Remote treatment delivery in response to the COVID-19 pandemic. Psychother Psychosom. 2020;89(3):130-2.

7 World Health Organization . Individual psychological help for adults impaired by distress in communities exposed to adversity (Generic field-trial version 1.0). Geneva: WHO; 2016.

8 Dawson KS, Bryant RA, Harper M, Kuowei Tay A, Rahman A, Schafer A, et al. Problem Management Plus (PM+): a WHO transdiagnostic psychological intervention for common mental health problems. World Psychiatry. 2015;14(3):354-7.

9 Rahman A, Hamdani SU, Awan NR, Bryant RA, Dawson KS, Khan MF, et al. Effect of a multicomponent behavioral intervention in adults impaired by psychological distress in a conflict-affected area of Pakistan: a randomized clinical trial. JAMA. 2016;316(24):260917.

10 Bryant RA, Schafer A, Dawson KS, Anjuri D, Mulili C, Ndogoni L, et al. Effectiveness of a brief behavioural intervention on psychological distress among women with a history of gender-based violence in urban Kenya: a randomised clinical trial. PLoS Med. 2017;14(8): e1002371.

11 Rahman A, Khan MN, Hamdani SU, Chiumento A, Akhtar P, Nazir H, et al. Effectiveness of a brief group psychological intervention for women in a post-conflict setting in Pakistan: a cluster randomized controlled trial. Lancet. 2019;393:1733-44.

12 Keyan D, Dawson K, Azevado S, Yadav S, Tran J, Bryant RA. Brief videoconferencing psychological intervention for reducing $\mathrm{CO}$ VID-19 related distress: study protocol for a randomized controlled trial. BMC Public Health. 2021;21(1):474.

13 Boutron I, Moher D, Altman DG, Schulz KF, Ravaud P. Extending the CONSORT statement to randomized trials of nonpharmacologic treatment: explanation and elaboration. Ann Intern Med. 2008;148(4):295-309.

14 Goldberg D, Williams PA. User's guide to the general health questionnaire. Windsor: NFER; 1988.

15 Minhas F, Mubbashar M. Validation of general health questionnaire (GHQ-12) in primary care settings of Pakistan. J Coll Phys Surg Pakistan. 1996;6:133-6.

16 Guidi J, Brakemeier EL, Bockting CLH, Cosci F, Cuijpers P, Jarrett RB, et al. Methodological recommendations for trials of psychological interventions. Psychother Psychosom. 2018; 87(5):276-84.
17 Zigmond AS, Snaith RP. The hospital anxiety and depression scale. Acta Psychiatr Scand. 1983;67(6):361-70.

18 Svindseth MF, Nøttestad JA, Dahl AA. A study of outcome in patients treated at a psychiatric emergency unit. Nord J Psychiatry. 2010;64(6):363-71.

19 Bjelland I, Dahl AA, Tandgen Haug T, Neckelmann D. The validity of the hosptialhospital and depression scale: an updated literature review. J Psychosom Res. 2002;52:69-77.

20 Stern AF. The hospital anxiety and depression scale. Occup Med. 2014;64(5):393-4.

21 Spitzer RL, Kroenke K, Williams JB, Löwe B. A brief measure for assessing generalized anxiety disorder: the GAD-7. Arch Intern Med. 2006;166(10):1092-7.

22 Toussaint A, Hüsing P, Gumz A, Wingenfeld K, Härter M, Schramm E, et al. Sensitivity to change and minimal clinically important difference of the 7-item Generalized Anxiety Disorder Questionnaire (GAD-7). J Affect Disord. 2020;265:395-401.

23 Morin CM, Stone J, McDonald K, Jones S. Psychological management of insomnia: a clinical replication series with 100 patients. Behavior Therapy. 1994;25(2):291-309.

24 Bastien CH, Vallières A, Morin CM. Validation of the Insomnia Severity Index as an outcome measure for insomnia research. Sleep Med. 2001;2:297-307.

25 Morin CM, Belleville G, Bélanger L, Ivers H. The Insomnia Severity Index: psychometric indicators to detect insomnia cases and evaluate treatment response. Sleep. 2011;34(5): 601-8.

26 Watson D, Clark LA, Tellegen A. Development and validation of brief measures of positive and negative affect: the PANAS scales. J Pers Soc Psychol. 1988;54(6):1063-70.

27 MacDowell SG, Wellons R, Bissell A, Knecht L, Naquin C, Karpinski A. The impact of symptoms of anxiety and depression on subjective and objective outcome measures in individuals with vestibular disorders. J Vestib Res. 2018;27(5-6):295-303.

28 Fawcett J, Clark DC, Scheftner WA, Gibbons RD. Assessing anhedonia in psychiatric patients. Arch Gen Psychiatry. 1983;40(1):7984.

29 Taylor S, Landry CA, Paluszek MM, Fergus TA, McKay D, Asmundson GJG. Development and initial validation of the COVID Stress Scales. J Anxiety Disord. 2020;72: 102232 .
30 Raudenbush SW. Comparing personal trajectories and drawing causal inferences from longitudinal data. Annu Rev Psychol. 2001; 52:501-25.

31 Norman GR, Sloan JA, Wyrwich KW. Interpretation of changes in health-related quality of life: the remarkable universality of half a standard deviation. Med Care. 2003;41(5): 582-92.

32 Santabarbara J, Lasheras I, Lipnicki DM, Bueno-Notivol J, Perez-Moreno M, Lopez-Anton $\mathrm{R}$, et al. Prevalence of anxiety in the COVID-19 pandemic: an updated meta-analysis of community-based studies. Prog Neuropsychopharmacol Biol Psychiatry. 2020;109: 110207.

33 Kauffman CD, Reist C, Djenderedjian A, Nelson JN, Haier RJ. Biological markers of affective disorders and posttraumatic stress disorder: a pilot study with desipramine. J Clin Psychiatry. 1987;48(9):366-7.

34 Breslau J, Finucane ML, Locker AR, Baird MD, Roth EA, Collins RL. A longitudinal study of psychological distress in the United States before and during the COVID-19 pandemic. Prev Med. 2021;143:106362.

35 Pan KY, Kok AAL, Eikelenboom M, Horsfall M, Jörg F, Luteijn RA, et al. The mental health impact of the COVID-19 pandemic on people with and without depressive, anxiety, or obsessive-compulsive disorders: a longitudinal study of three Dutch case-control cohorts. Lancet Psychiatry. 2021;8(2):121-9.

36 Wahlund T, Mataix-Cols D, Olofsdotter Lauri K, de Schipper E, Ljótsson B, Aspvall K, et al. Brief online cognitive behavioural intervention for dysfunctional worry related to the COVID-19 pandemic: a randomised controlled trial. Psychother Psychosom. 2021; 90(3):191-9.

37 Reins JA, Buntrock C, Zimmermann J, Grund S, Harrer M, Lehr D, et al. Efficacy and moderators of internet-based interventions in adults with subthreshold depression: an individual participant data meta-analysis of randomized controlled trials. Psychother Psychosom. 2021;90(2):94-106.

38 Cosci F, Guidi J. The role of illness behavior in the COVID-19 pandemic. Psychother Psychosom. 2021;90(3):156-9.

39 Ganson KT, Weiser SD, Tsai AC, Nagata JM. Associations between anxiety and depression symptoms and medical care avoidance during COVID-19. J Gen Intern Med. 2020;35(11): 3406-8.

40 Chen JA, Zhang E, Liu CH. Potential impact of COVID-19-related racial discrimination on the health of Asian Americans. Am J Public Health. 2020;110(11):1624-7. 